\title{
Josephson junctions in thin and narrow rectangular superconducting strips
}

\author{
John R. Clem \\ Ames Laboratory and Department of Physics and Astronomy, \\ Iowa State University, Ames, Iowa, 50011-3160
}

(Dated: November 16, 2018)

\begin{abstract}
I consider a Josephson junction crossing the middle of a thin rectangular superconducting strip of length $L$ and width $W$ subjected to a perpendicular magnetic induction $B$. I calculate the spatial dependence of the gauge-invariant phase difference across the junction and the resulting $B$ dependence of the critical current $I_{c}(B)$.
\end{abstract}

PACS numbers: 74.50.+r,74.78.-w,74.25.-q,74.78.Na

\section{INTRODUCTION}

Grain-boundary Josephson junctions play an important role in thin films of $\mathrm{YBa}_{2} \mathrm{Cu}_{3} \mathrm{O}_{7-\delta}$ (YBCO). ${ }^{1-4}$ Various theoretical approaches have been taken to understand the physics of Josephson junctions in thin films. ${ }^{5-7}$ When the film thickness $d$ is less than the London penetration depth $\lambda$, the current density $\boldsymbol{j}$ is practically uniform across the thickness, and the characteristic length governing the spatial distribution of the magnetic field distribution is the Pearl length, ${ }^{8}$

$$
\Lambda=2 \lambda^{2} / d .
$$

However, various studies have shown that there is a nonlocal relationship between the Josephson-current distribution in the vicinity of a Josephson vortex core and the magnetic field these currents generate, ${ }^{9-12}$ and the characteristic length describing the spatial variation of the gauge-invariant phase across the junction is (in SI units)

$$
\ell=\phi_{0} / 4 \pi \mu_{0} \lambda^{2} j_{c},
$$

where $\phi_{0}=h / 2 e$ is the superconducting flux quantum and $j_{c}$ (assumed to be independent of position) is the maximum Josephson current density that can flow as a supercurrent through the junction.

The integral equations relating the gauge-invariant phase difference across the junction to the magnetic field generated by a vortex in a long Josephson junction in a thin $(d<\lambda)$ film of lateral dimensions large by comparison with $\Lambda$ and $\ell$ were examined analytically and solved numerically in Ref. 6 for arbitrary ratios of $\ell / \Lambda$. The case of a short Josephson junction bisecting a long superconducting strip of width $W$ was studied in Ref. 7 under the assumptions that $W \ll \Lambda$ and $W \ll \ell$.

In this paper I revisit the latter problem by considering a thin rectangular uniform superconducting strip of length $L$, width $W$, and thickness $d(d<\lambda)$ divided into two halves by a Josephson junction at $x=0$, as shown in Fig. 1. An applied magnetic induction $\boldsymbol{B}=\hat{z} B$ induces screening currents in the film. However, I consider here only the simplest case for which $\Lambda=2 \lambda^{2} / d$ is much larger than the smaller of $L$ and $W$, such that the self-field generated by the screening currents can be neglected. ${ }^{7}$ The purpose of this paper is to calculate how the screening

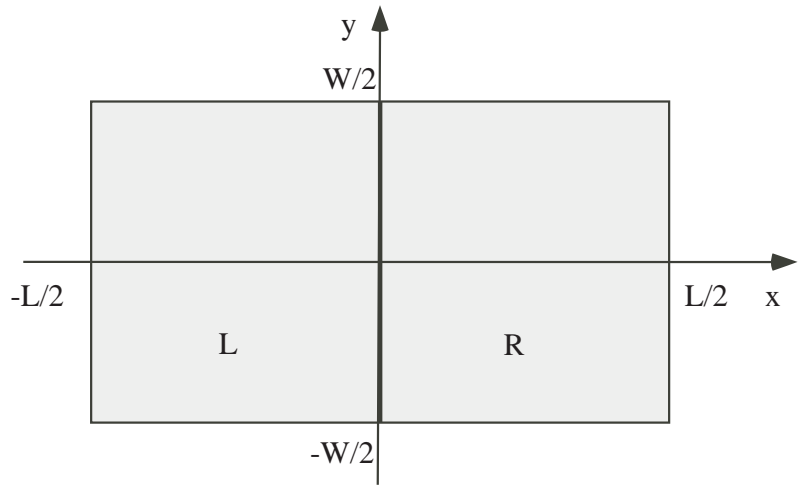

FIG. 1: Sketch of the thin rectangular superconducting strip of width $W$ and length $L$ considered here. An in-line Josephson junction of width $d_{i}$ (bold line) is at the center at $x=0$. A magnetic induction $B$ is applied in the $z$ direction. Current leads of separation $L$ (not shown) symmetrically feed current $I$ to the sample along the $x$ direction.

currents induced in response to $\boldsymbol{B}$ affect the $B$ dependence of the maximum Josephson critical current $I_{c}(B)$.

In Sec. II I give a brief discussion of the derivation of the basic equation for the gauge-invariant phase difference $\Delta \gamma(y)$ across the junction, in Sec. III I present the solutions for $\Delta \gamma(y)$ for arbitrary ratios of $L / W$, and in Sec. IV I briefly summarize the results.

\section{GAUGE-INVARIANT PHASE DIFFERENCE}

In the context of the Ginzburg-Landau (GL) theory, ${ }^{13,14}$ the superconducting order parameter can be expressed as $\Psi=\Psi_{0} f e^{i \gamma}$, where $\Psi_{0}$ is the magnitude of the order parameter in equilibrium, $f=|\Psi| / \Psi_{0}$ is the reduced order parameter, and $\gamma$ is the phase. The second GL equation (in SI units) is

$$
\boldsymbol{j}=-\frac{f^{2}}{\mu_{0} \lambda^{2}}\left(\boldsymbol{A}+\frac{\phi_{0}}{2 \pi} \nabla \gamma\right)
$$

where $\boldsymbol{A}$ is the vector potential and $\boldsymbol{B}=\nabla \times \boldsymbol{A}$ is the magnetic induction. Since $\boldsymbol{j}$ is a gauge-invariant quantity, so is the quantity within the parentheses on the 
right-hand side. Different choices for the gauge of the vector potential $\boldsymbol{A}$ result in different expressions for $\gamma$.

With a sinusoidal current-phase relation, the Josephson current density in the $x$ direction across the junction of width $d_{i}$ at $x=0$ is $j_{x}(y)=j_{c} \sin \Delta \gamma(y)$, where $j_{c}$ is the maximum Josephson current density and $\Delta \gamma(y)$ is the gauge-invariant phase difference between the left (a) and right (b) superconductors,

$$
\Delta \gamma(y)=\gamma_{a}\left(-\frac{d_{i}}{2}, y\right)-\gamma_{b}\left(\frac{d_{i}}{2}, y\right)-\frac{2 \pi}{\phi_{0}} \int_{-d_{i} / 2}^{d_{i} / 2} A_{x}(x, y) d x
$$

I assume here that the induced or applied current densities $\boldsymbol{j}_{\mathrm{a}}$ and $\boldsymbol{j}_{\mathrm{b}}$ on the left- and right-hand sides of the junction are so weak that the suppression of the magnitude of the superconducting order parameter is negligible, such that $f=1$. A simple relation between these current densities and the gauge-invariant phase difference can be obtained by integrating the vector potential $\boldsymbol{A}$ around a very narrow rectangular loop of width $d_{i}$ in the $x y$ plane that just encloses the junction (with the bottom end at the origin and the top end at $y$ ), neglecting the magnetic flux up through the contour, making use of Eq. (3) with $f=1$ for those portions of the integration along the sides of the junction, and noting that, by symmetry, $j_{\mathrm{a} y}(0, y)=-j_{\mathrm{b} y}(0, y)$ :

$$
\Delta \gamma(y)=\Delta \gamma_{0}+\frac{4 \pi \mu_{0} \lambda^{2}}{\phi_{0}} \int_{0}^{y} j_{\mathrm{b} y}\left(0, y^{\prime}\right) d y^{\prime}
$$

where $\Delta \gamma_{0}=\Delta \gamma(0)$, such that

$$
d \Delta \gamma(y) / d y=\left(4 \pi \mu_{0} \lambda^{2} / \phi_{0}\right) j_{\mathrm{b} y}(0, y) .
$$

\section{SOLVING FOR $\Delta \gamma$}

I next assume that the Josephson coupling is so weak that the currents $\boldsymbol{j}_{\mathrm{a}}$ and $\boldsymbol{j}_{\mathrm{b}}$ on the left- and right-hand sides of the junction induced in response to the applied magnetic induction $\boldsymbol{B}$ are far larger than the Josephson current density. This is equivalent to the assumption that $W \ll \ell$. Since $\boldsymbol{j}_{\mathrm{a}}$ easily can be obtained by symmetry from $\boldsymbol{j}_{\mathrm{b}}$, I calculate only $\boldsymbol{j}_{\mathrm{b}}$ in the region $x>0$ and suppress the subscript b.

With the gauge choice $\boldsymbol{A}=-\hat{x} B y$, since $\nabla \cdot \boldsymbol{j}=0$ [see Eq. (3)], $\nabla^{2} \gamma=0$ must be solved subject to the boundary conditions following from $j_{x}(0, y)=j_{x}(L / 2, y)=0$ and $j_{y}(x, \pm W / 2)=0$,

$$
\begin{aligned}
& \gamma_{x}(0, y)=\gamma_{x}(L / 2, y)=2 \pi B y / \phi_{0}, \\
& \gamma_{y}(x, \pm W / 2)=0,
\end{aligned}
$$

where $\gamma_{x}=\partial \gamma / \partial x$ and $\gamma_{y}=\partial \gamma / \partial y$. The solution, obtained by the method of separation of variables, is (up to a constant)

$$
\gamma(x, y)=\frac{8 \pi B}{\phi_{0} W} \sum_{n=0}^{\infty} \frac{(-1)^{n} \sinh \left[k_{n}(x-L / 4)\right] \sin \left(k_{n} y\right)}{k_{n}^{3} \cosh \left(k_{n} L / 4\right)},
$$

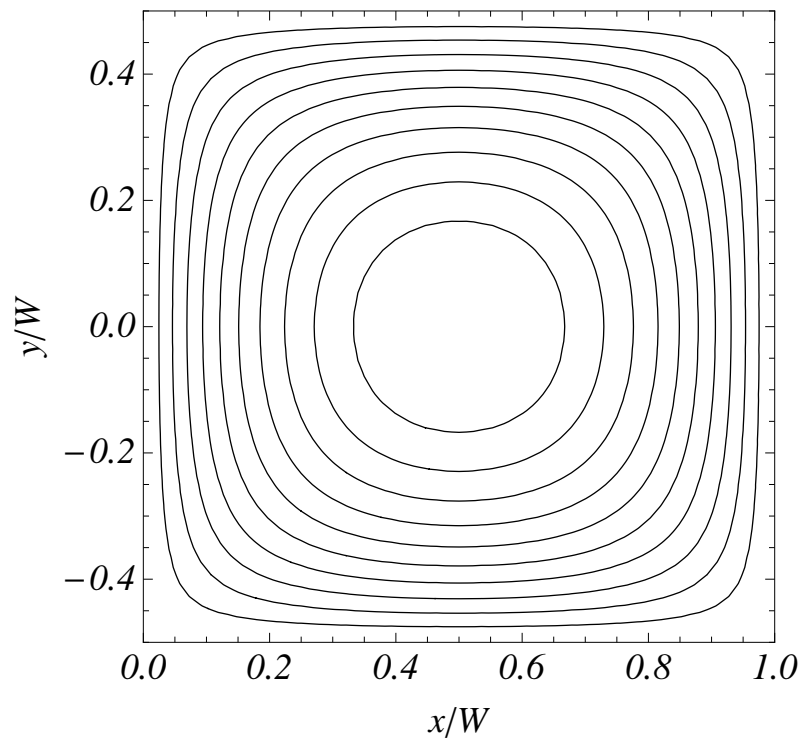

FIG. 2: Contour plot of the stream function $S(x, y)$ in the right half $(x>0)$ of a strip of length $L=2 W$. The contours correspond to the streamlines of the current density $\boldsymbol{j}$ induced by the applied magnetic induction $\boldsymbol{B}=\hat{z} B$.

where $k_{n}=(n+1 / 2) 2 \pi / W$.

The current density $\boldsymbol{j}(x, y)$ now can be obtained from Eq. (3). Since $\nabla \cdot \boldsymbol{j}=0$, we also can write $\boldsymbol{j}=\nabla \times \boldsymbol{S}$, where $\boldsymbol{S}=\hat{z} S$, and $S(x, y)=\left(B / 2 \mu_{0} \lambda^{2}\right) s(x, y)$ is the stream function given by

$s(x, y)=y^{2}+\frac{8}{W} \sum_{n=0}^{\infty} \frac{(-1)^{n} \cosh \left[k_{n}(x-L / 4)\right] \cos \left(k_{n} y\right)}{k_{n}^{3} \cosh \left(k_{n} L / 4\right)}$.

The series converges rapidly, and Fig. 2 shows the result for $x>0$ obtained by summing over $n$ from 0 to 10 when $L / W=2$.

Since Eq. (3) yields $j_{y}(0, y)=-\left(\phi_{0} / 2 \pi \mu_{0} \lambda^{2}\right) \gamma_{y}(0, y)$, Eq. (6) can be integrated to obtain (assuming the additive constant $\Delta \gamma_{0}=0$ )

$$
\Delta \gamma(y)=\frac{16 \pi B}{\phi_{0} W} \sum_{n=0}^{\infty} \frac{(-1)^{n}}{k_{n}^{3}} \tanh \left(k_{n} L / 4\right) \sin \left(k_{n} y\right) .
$$

As shown in the Appendix, when $L \rightarrow \infty$, the sum can be expressed in terms of the Lerch transcendent $\Phi(z, s, a)$, and in this limit $\Delta \gamma(y)$ also can be expressed as $\Delta \gamma(y)=$ $\left(4 B W^{2} / \phi_{0}\right) \varphi_{0}(\pi y / W)$, where $\varphi_{0}(\mu)$ is a function defined in Ref. 7. This is the explanation of why $\varphi_{0}(\mu)$ was found to be a material-independent universal function ${ }^{7}$ when $\Lambda \gg W$ and $\ell \gg W$.

For all ratios of $L / W$, the maximum value of $\Delta \gamma(y)$ occurs at $y=W / 2$, where

$$
\begin{aligned}
& \Delta \gamma(W / 2)=\frac{14 \zeta(3) B W^{2}}{\pi^{2} \phi_{0}}=1.705 \frac{B W^{2}}{\phi_{0}}, L \rightarrow \infty, \\
& \Delta \gamma(W / 2)=\frac{\pi B W L}{2 \phi_{0}}=1.571 \frac{B W L}{\phi_{0}}, L \ll W .
\end{aligned}
$$




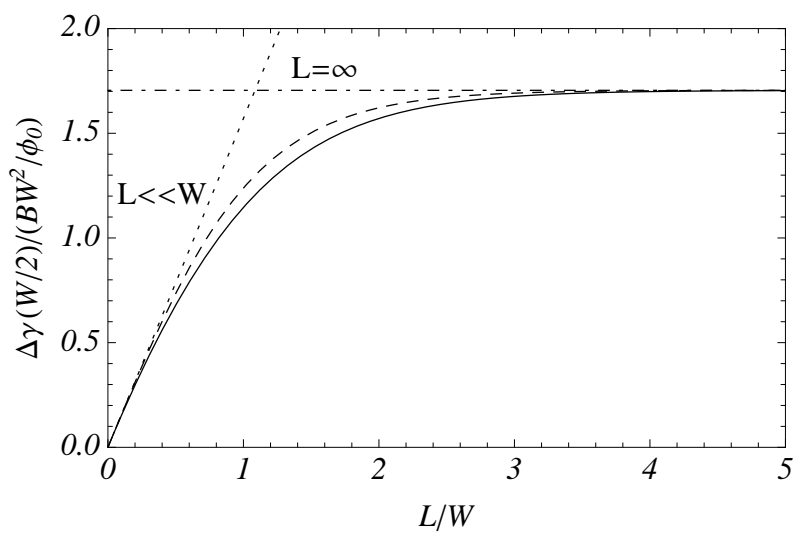

FIG. 3: $\Delta \gamma(W / 2)$ (solid) vs $L / W$ and the approximations of Eqs. (12) (dot-dashed), (13) (dotted), and (14) (dashed).

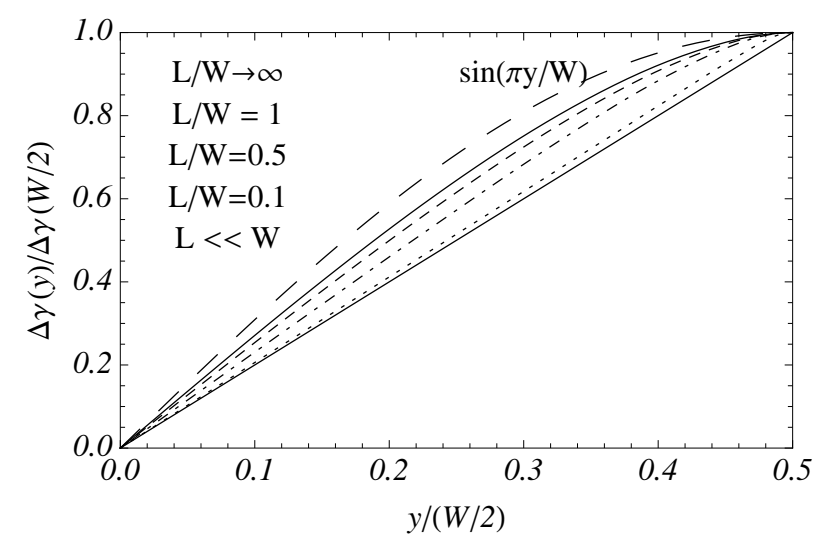

FIG. 4: $\Delta \gamma(y) / \Delta \gamma(W / 2)$ vs $y /(W / 2)$ for $L \rightarrow \infty$ (upper solid curve), $L / W=1$ (dashed), $L / W=0.5$ (dot-dashed), $L / W=0.1$ (dotted), and $L \ll W$ (lower solid curve), for which $\Delta \gamma(y) / \Delta \gamma(W / 2)=y /(W / 2)$. For comparison, the top long-dashed curve shows $\sin (\pi y / W)$.

The solid curve in Fig. 3 shows $\Delta \gamma(W / 2)$ normalized to $B W^{2} / \phi_{0}$ as a function of $L / W$ along with the limiting behaviors of Eqs. (12) (dot-dashed) and (13) (dotted). The dashed curve shows the interpolating function,

$$
\Delta \gamma(W / 2)=\frac{14 \zeta(3) B W^{2}}{\pi^{2} \phi_{0}} \tanh \left[\frac{\pi^{3} L}{28 \zeta(3) W}\right]
$$

where $\zeta(3)=1.20206$ is the Riemann zeta function.

The plots of $\Delta \gamma(y) / \Delta \gamma(W / 2)$ vs $y /(W / 2)$ in Fig. 4 show how the gauge-invariant phase difference depends upon the ratio $L / W$. For $L / W \rightarrow \infty$, the curve lies below $\sin (\pi y / W)$, shown as the long-dashed curve, and for $L \ll W, \Delta \gamma(y) / \Delta \gamma(W / 2)=y /(W / 2)$, a straight line.

The maximum Josephson current $I_{c}(B)$, the maximum integral of $j_{c} d \sin \Delta \gamma(y)$ over $y$ from $-W / 2$ to $W / 2$, occurs when $\Delta \gamma_{0}= \pm \pi / 2$, such that

$$
\frac{I_{c}(B)}{I_{c}(0)}=\frac{2}{W}\left|\int_{0}^{W / 2} \cos [\Delta \gamma(y)] d y\right|
$$

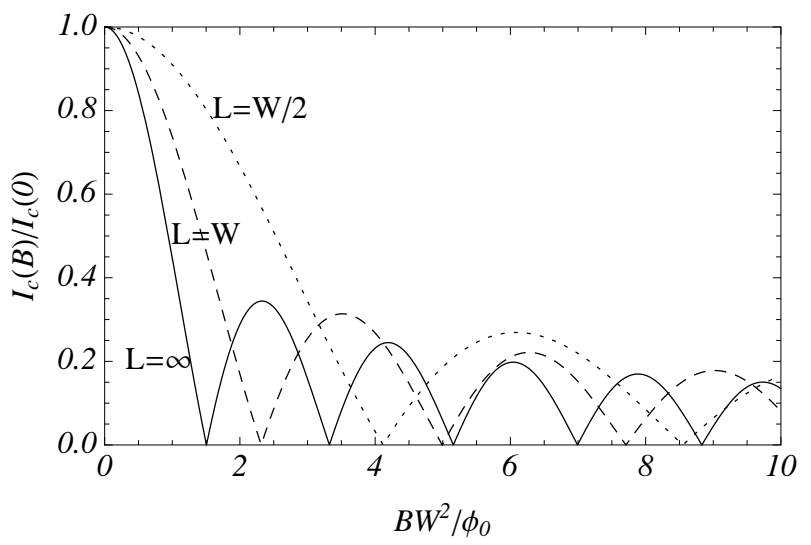

FIG. 5: $I_{c}(B) / I_{c}(0)$ vs $B W^{2} / \phi_{0}$ calculated from Eqs. (11) and (15) for $L / W=\infty$ (solid), 1 (dashed) and $1 / 2$ (dotted).

where $\Delta \gamma(y)$ is given in Eq. (11). Figure 5 shows plots of $I_{c}(B) / I_{c}(0)$ vs $B W^{2} / \phi_{0}$ for $L / W=\infty, 1$, and $1 / 2$. The stretching out of the pattern along the horizontal axis as $L$ decreases is easily understood with the help of Fig. 3.

Let us define $\Delta B_{1}$ as the value of $B$ at which $I_{c}(B)$ has its first zero, $\Delta B_{2}$ as the difference of the values at which $I_{c}(B)$ has its second and first zeros, and $\Delta B_{n}$ as the difference of the values at which $I_{c}(B)$ has its $n$th and $(n-1)$ th zeros. For all finite values of $L / W$, the $\Delta B_{n}$ are smaller for small $n$ than for large $n$. However, for large $n$, the $\Delta B_{n}$ approach the limiting value

$$
\Delta B=\left(\frac{\pi^{3} \phi_{0}}{16 W^{2}}\right) / \sum_{n=0}^{\infty} \frac{\tanh [(2 n+1) \pi L / 4 W]}{(2 n+1)^{3}} .
$$

To illustrate this, if we approximate $\Delta \gamma(y)$ for $L \rightarrow \infty$ by $\Delta \gamma(W / 2) \sin (\pi y / W)$ (see the long-dashed curve in Fig. 4), as in Ref. 7, then the integral in Eq. 15 can be evaluated in terms of the Bessel function $J_{0}$ with the result

$$
\frac{I_{c}(B)}{I_{c}(0)}=\left|J_{0}\left(\frac{14 \zeta(3) B W^{2}}{\pi^{2} \phi_{0}}\right)\right|
$$

For $L / W \rightarrow \infty$, the sum in Eq. (16) can be evaluated as

$$
\Delta B=\left[\pi^{3} / 14 \zeta(3)\right] \phi_{0} / W^{2}=1.842 \phi_{0} / W^{2},
$$

as pointed out in Ref. 15. Using Eq. (17) and the wellknown zeros of $J_{0}(x)$, we find the following values for $n$ $=1,2,3,4$, and $5: \Delta B_{n} / \Delta B=0.7655,0.9916,0.9975$, 0.9988 , and 0.9993. However, to evaluate the zeros of $I c(B) / I_{c}(0)$ without using the Bessel-function approximation, we must numerically evaluate Eq. (15). This yields the following more accurate values for $n=1,2$, 3, 4, and 5: $\Delta B_{n} / \Delta B=0.8173,0.9866,0.9946,0.9968$, and 0.9979 .

When $d_{i} \ll L \ll W$, the gauge-invariant phase difference $\Delta \gamma(y)$ of Eq. (11) becomes linear in $y$, and in this case we have the familiar Fraunhofer-like pattern,

$$
\frac{I_{c}(B)}{I_{c}(0)}=\left|\frac{\sin \left(\pi B W L / 2 \phi_{0}\right)}{\pi B W L / 2 \phi_{0}}\right|,
$$




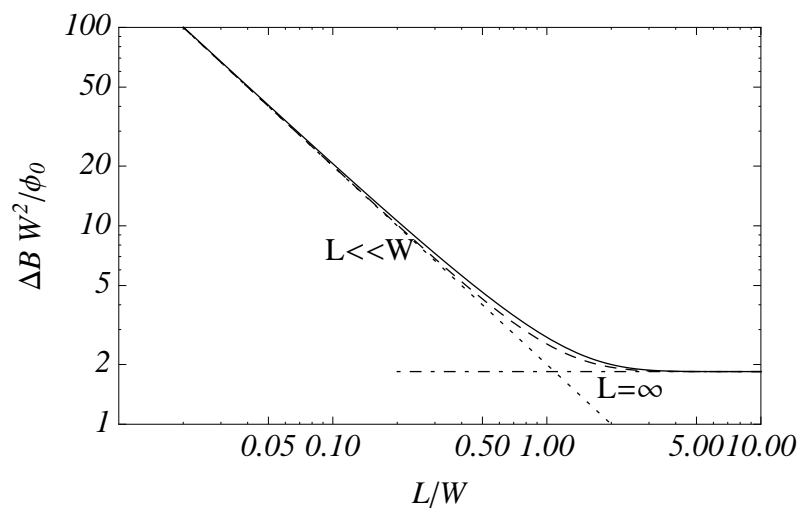

FIG. 6: $\Delta B$ vs $L / W$ calculated from Eqs. (16) (solid), (18) (dot-dashed), (20) (dotted), and (21) (dashed).

such that all the $\Delta B_{n}$ are the same and equal to

$$
\Delta B=2 \phi_{0} / W L .
$$

The magnitude of this $\Delta B$ agrees with that in sandwichtype Josephson junctions with thickness $d \gg \lambda$ along the $z$ direction only in the limit $d_{i} \ll L<\lambda{ }^{16-18}$

The solid curve in Fig. 6 shows $\Delta B$, the large- $n$ limit of $\Delta B_{n}$, calculated via Eq. (16) as a function of $L / W$, along with the expressions for $\Delta B$ in the limits $L / W \rightarrow \infty$ (dot-dashed) and $L \ll W$ (dotted). The dashed curve shows the approximate interpolating function obtained from Eq. (14),

$$
\Delta B=\left(\frac{\pi^{3} \phi_{0}}{14 \zeta(3) W^{2}}\right) / \tanh \left[\frac{\pi^{3} L}{28 \zeta(3) W}\right] .
$$

\section{SUMMARY}

In this paper I have considered a Josephson junction bisecting a rectangular superconducting thin film of large Pearl length $\Lambda=2 \lambda^{2} / d$ subjected to a perpendicular magnetic induction $B$. I calculated the gauge-invariant phase difference and used it to determine the $B$ dependence of the Josephson critical current density $I_{c}(B)$.

\section{Acknowledgments}

I thank J. E. Sadleir and V. G. Kogan for stimulating discussions. My work at the Ames Laboratory was supported by the Department of Energy - Basic Energy Sciences under Contract No. DE-AC02-07CH11358.

\section{Appendix A: The limit $L \rightarrow \infty$}

In the limit $L \rightarrow \infty$, the gauge-invariant phase difference $\Delta \gamma(y)$ given in Eq. (11) can be expressed as $\Delta \gamma(y)=\left(16 B W^{2} / \pi^{2} \phi_{0}\right) \sigma(\pi y / W)$, where

$$
\begin{aligned}
\sigma(\psi)= & \sum_{n=0}^{\infty}(-1)^{n} \sin [(2 n+1) \psi] /(2 n+1)^{3} \\
= & (i / 16) e^{-i \psi}\left[\Phi\left(-e^{-2 i \psi}, 3,1 / 2\right)\right. \\
& \left.-e^{2 i \psi} \Phi\left(-e^{2 i \psi}, 3,1 / 2\right)\right]
\end{aligned}
$$

and $\Phi(z, s, a)=\sum_{k=0}^{\infty} z^{k} /(k+a)^{s}$ is the Lerch transcendent. ${ }^{19}$ Note that $\sigma(0)=0$ and $\sigma(\pi / 2)=$ $7 \zeta(3) / 8=1.0518$.
1 D. J. Van Harlingen, Rev. Mod. Phys. bf 67, 515 (1995).

${ }^{2}$ K. A. Delin and A. W. Kleinsasser, Supercond. Sci. Technol. 9, 227 (1996).

${ }^{3}$ H. Hilgenkamp and J. Mannhart, Rev. Mod. Phys. , 74, 485 (2002).

4 J. H. Durrell and N. A. Rutter, Supercond. Sci. Technol. 22, 013001 (2009).

${ }^{5}$ R. G. Mints and I. B. Snapiro, Phys. Rev. B 49, 6188 (1994).

${ }^{6}$ V. G. Kogan, V. V. Dobrovitski, J. R. Clem, Y. Mawatari, and R. G. Mints, Phys. Rev. B 63, 144501 (2001)

7 M. Moshe, V. G. Kogan, and R. G. Mints, Phys. Rev. B 78, 020510(R) (2008).

8 J. Pearl, Appl. Phys. Lett. 5, 65 (1964).

9 Yu. M. Ivanchenko and T. K. Soboleva, Phys. Lett. A bf 147, 65 (1990).

10 A. Gurevich, Phys. Rev. B 46, 3187 (1992).
11 Yu. M. Ivanchenko, Phys. Rev. B 52, 79 (1995).

12 Yu. E. Kuzovlev and A. I. Lomtev, Sov. Phys. JETP 84, 986 (1997).

13 P. G. de Gennes, Superconductivity of Metals and Alloys (Benjamin, New York, 1966), p. 177.

14 D. Saint-James, E. J. Thomas, and G. Sarma, Type II Superconductivity (Pergamon, Oxford, 1969).

15 P. A. Rosenthal, M. R. Beasley, K. Char, M. S. Colclough, and G. Zaharchuk, Appl. Phys. Lett. 59, 3482 (1991).

16 M. Weihnacht, Phys. Stat. Sol. 32, K169 (1969).

17 A. Barone and G. Paterno, Physics and Applications of the Josephson Effect, (Wiley, New York, 1982).

18 T. P. Orlando and K. A. Delin, Foundations of Applied Superconductivity, (Addison-Wesley, Reading, 1991), p. 447.

19 Wolfram Research, Inc., Mathematica, Version 7.0, Champaign, IL (2008). 